\title{
The cooling of coronal plasmas
}

\section{Properties of the radiative phase}

\author{
S. J. Bradshaw and P. J. Cargill
}

\begin{abstract}
Space and Atmospheric Physics, Blackett Laboratory, Imperial College London, Prince Consort Road, London, SW7 2BZ, UK
\end{abstract} e-mail: s.bradshaw@imperial.ac.uk

Received 22 November 2004 / Accepted 16 March 2005

\begin{abstract}
The robust $T \propto n^{\delta}$ power-law relationship between the temperature $(T)$ and the density $(n)$ that arises during the radiative cooling phase of a solar coronal loop is investigated. Using an analytical model and numerical hydrodynamic simulations, we demonstrate that radiative energy loss from the transition region is the dominant physical process. It governs the down-flow by which mass is lost from the corona and hence controls the evolution of the entire loop.

We also show that the down-flow is initiated by a weakening of the pressure gradient between the corona and the transition region, such that the plasma can no longer be supported in hydrostatic equilibrium. Rather than driving the down-flow, the pressure gradient actually regulates it and acts as a brake against gravitational acceleration.
\end{abstract}

Key words. Sun: corona - Sun: transition region - Sun: UV radiation

\section{Introduction}

It is commonly thought that much of the energy deposited in the solar corona occurs in very impulsive bursts of energy dissipation lasting a few seconds or minutes. While solar flares are the largest type of such energy release, it is plausible that bursty heating can occur over at least nine orders of magnitude from $10^{24}$ (nanoflares) up to $10^{33} \mathrm{ergs}$ (large flares). Unfortunately, because of its short duration, impulsive heating is often very difficult to detect directly, especially at the lower end of the energy scale, and so any observed signature comes largely from cooling coronal plasma.

Impulsively-heated coronal plasmas cool by both thermal conduction and radiation, and satisfy an energy equation:

$\frac{\mathrm{d} P}{\mathrm{~d} t}=-\gamma P \frac{\partial V}{\partial s}+(\gamma-1)\left[\frac{\partial}{\partial s}\left(\kappa_{0} T^{5 / 2} \frac{\partial T}{\partial s}\right)-E_{\mathrm{rad}}\right]$,

in the usual notation. To order of magnitude, a loop of length $L$ will cool by conduction and radiation on time-scales:

$\tau_{\mathrm{c}}=\frac{3 n k L^{2}}{\kappa_{0} T^{5 / 2}}, \tau_{r}=\frac{3 k T^{1-\alpha}}{\chi^{n}}$,

where we have assumed that $E_{\mathrm{rad}}=n^{2} \Lambda(T)=n^{2} \chi T^{\alpha}$ (e.g. Priest 1982), $P=2 n k_{\mathrm{B}} T$, and $\gamma=\frac{5}{3}$. Conductive cooling is important at high temperatures and low densities, whereas radiation is more important at lower temperatures and higher densities. This paper is concerned with radiative cooling.

It is well known that when a coronal plasma cools through a series of equilibria, the density scales approximately as $T^{2}$ (e.g. Rosner et al. 1978). Plasma must also drain from the loop during this cooling. An example of such a situation is when the coronal energy input decreases on a time-scale much longer than $\tau_{\mathrm{c}}$ or $\tau_{r}$. However, on the basis of numerical simulations and observations of solar flares Serio et al. (1991) and Jakimiec et al. (1992) noted that during the radiative cooling phase of flares the temperature and density were related by a power-law of the form:

$T \propto n^{\delta}$,

where $\delta \approx 2$. Subsequent investigations by Sylwester et al. (1993) and Cargill et al. (1995, hereafter CMA95) showed that while $\delta$ was not always two, the generic power-law relationship was very robust.

The difference between these two approaches to radiative cooling are significant. Consider a coronal plasma that cools from temperature $T_{0}$ to temperature $T_{1}$. Then the ratio of the density obtained from a radiative model described by Eq. (3) $\left(n_{r}\right)$ to that from an equilibrium model $\left(n_{\mathrm{eq}}\right)$ is $n_{r} / n_{\mathrm{eq}}=\left(T_{0} / T_{1}\right)^{3 / 2}$. So the radiative model gives an "overdense" loop since it has a higher density than the equilibrium model (e.g. Cargill \& Klimchuk 2004). Since the coronal emission measure scales as $n^{2}$, the effect on the observed radiative signature can be large. Despite this higher density, one would expect the magnitude of the velocity associated with the loop draining to be larger for the case of uncompensated radiative cooling simply because the cooling time-scale is much shorter $\left(\tau_{r}\right.$, rather than the time-scale for the equilibrium to evolve slowly).

The scalings between temperature and density described in Eq. (3) have been used by Cargill $(1993,1994)$ and CMA95 to 
develop a series of simple analytical models of the cooling rate of impulsively heated coronal plasmas that could also be applied to studies of stellar flares. However, despite their ready appearance in numerical simulations, the cause of these robust $n-T$ scalings has never been established. We return to this problem from the viewpoint of analytical models and numerical simulations.

In Sect. 2 we develop an analytical model that constrains $\delta$ by considering the interaction of the transition region with the corona. Section 3 contains results from numerical simulations of cooling loops and a comparison with our analytical results. In Sect. 4 we present an improvement to our analytical model, based upon the results in Sect. 3. We summarise our results and present our conclusions in Sect. 5.

\section{An analytical model for radiative cooling}

\subsection{The coronal region}

We develop a model of the coronal region during the radiative cooling phase, thereby assuming that thermal conduction in the corona can be neglected and the plasma down-flow associated with radiative cooling is already well-established. This downflow arises due to the fact that the radiative losses at the base of the loop are greater than at the apex, which reduces the pressure gradient that supports the plasma against gravity when the loop is in hydrostatic equilibrium. When the pressure gradient can no longer counteract the gravitational force, the down-flow begins to develop. Consequently, the coronal part of the loop loses energy both to space by optically thin radiation and to the cooler plasma further down, by convection.

Integrating Eq. (1) over a characteristic coronal lengthscale, $L_{\mathrm{c}}$, we obtain:

$\frac{\mathrm{d} P_{\mathrm{c}}}{\mathrm{d} t}=-\gamma \frac{P_{\mathrm{c}} V_{\mathrm{c}}}{L_{\mathrm{c}}}-(\gamma-1) n_{\mathrm{c}}^{2} \chi T_{\mathrm{c}}^{\alpha}$

where subscript $\mathrm{c}$ denotes coronal averaged quantities. Integrating the equation of mass conservation we find:

$\frac{\mathrm{d} n_{\mathrm{c}}}{\mathrm{d} t}=-\frac{n_{\mathrm{c}} V_{\mathrm{c}}}{L_{\mathrm{c}}}$

Combining Eqs. (4) and (5) gives:

$\frac{\mathrm{d} P_{\mathrm{c}}}{\mathrm{d} n_{\mathrm{c}}}=\frac{\gamma P_{\mathrm{c}}}{n_{\mathrm{c}}}+(\gamma-1) \frac{n_{\mathrm{c}} L_{\mathrm{c}} \chi T_{\mathrm{c}}^{\alpha}}{V_{\mathrm{c}}}$

We now write Eq. (3) in the form:

$\frac{T_{\mathrm{c}}}{T_{\mathrm{c} 0}}=\left(\frac{n_{\mathrm{c}}}{n_{\mathrm{c} 0}}\right)^{\delta}$

which implies:

$$
\frac{P_{\mathrm{c}}}{P_{\mathrm{c} 0}}=\left(\frac{n_{\mathrm{c}}}{n_{\mathrm{c} 0}}\right)^{\delta+1}
$$

where subscript 0 corresponds to the start of the radiative cooling phase. From Eq. (8):

$\frac{\partial P_{\mathrm{c}}}{\partial t}=(\delta+1) \frac{P_{\mathrm{c} 0}}{n_{\mathrm{c} 0}}\left(\frac{n_{\mathrm{c}}}{n_{\mathrm{c} 0}}\right)^{\delta} \frac{\partial n_{\mathrm{c}}}{\partial t}$ and, using Eq. (7), Eq. (6) becomes:

$\frac{P_{\mathrm{c} 0}}{n_{\mathrm{c} 0}}\left(\frac{n_{\mathrm{c}}}{n_{\mathrm{c} 0}}\right)^{\delta}(\delta+1-\gamma)=(\gamma-1) \chi n_{\mathrm{c} 0} T_{\mathrm{c} 0}^{\alpha} L_{\mathrm{c}}\left(\frac{n_{\mathrm{c}}}{n_{\mathrm{c} 0}}\right)^{\alpha \delta+1} V_{\mathrm{c}}^{-1}$.

The power of $n_{\mathrm{c}}$ must be the same on each side of Eq. (10). This is clearly not the case for common values of $\alpha$ and $\delta\left(-\frac{1}{2}\right.$ and 2, respectively: CMA95) which implies that $V_{\mathrm{c}}$ must scale as the coronal density to some power. On writing:

$V_{\mathrm{c}}=V_{\mathrm{c} 0}\left(\frac{n_{\mathrm{c}}}{n_{\mathrm{c} 0}}\right)^{\beta}$

we obtain from equating the power of $n_{\mathrm{c}}$ in Eq. (10):

$\beta=\delta(\alpha-1)+1$,

and from equating the coefficients:

$\delta=\gamma-1+\left(\frac{L_{\mathrm{c}}}{V_{\mathrm{c} 0}}\right)\left(\frac{(\gamma-1) \chi n_{\mathrm{c} 0}^{2} T_{\mathrm{c} 0}^{\alpha}}{P_{\mathrm{c} 0}}\right)=\frac{2}{3}+\left(\frac{\tau_{v 0}}{\tau_{r 0}}\right)$,

where $\tau_{v 0}=L_{\mathrm{c}} / V_{\mathrm{c} 0}, \tau_{r 0}$ is the initial radiative cooling time and we have set $\gamma=\frac{5}{3}$.

\subsection{Interaction with the transition region}

In order to progress further, we require a determination of $V_{\mathrm{c} 0}$. During the radiative cooling phase, any volumetric heating in the transition region is not sufficient to balance the large amount of energy lost through optically-thin radiation. Consequently, the transition region must receive a supply of energy from the corona. Since thermal conduction is negligible (by definition) during the radiative phase, the energy must be supplied by convection.

We now introduce a transition region into our analytical model, which we expect to provide us with new power-law relationships showing how its properties give rise to the power-law relationships for $T_{\mathrm{c}}$ and $V_{\mathrm{c}}$. The analytical scalings can then be checked by comparing the predicted values for the indices with those from full, numerical hydrodynamic simulations.

The equation of energy balance between the corona and the transition region is:

$-(\gamma+1) \frac{P_{\mathrm{c}} V_{\mathrm{c}}}{L_{\mathrm{t}}}=-(\gamma-1) n_{\mathrm{t}}^{2} \chi T_{\mathrm{t}}^{\alpha}$,

where subscript $\mathrm{t}$ denotes a transition region quantity. Equation (14) is essentially a statement of Gauss' Law applied to an energy flux across a surface - in this case across the interface between the corona and the transition region. Here, we assume that the energy provided to the transition region by the corona is radiated away to space. then:

Let $\Gamma=-(\gamma+1) /(\gamma-1)$ and, since $n_{\mathrm{t}}^{2} T_{\mathrm{t}}^{\alpha}=T_{\mathrm{t}}^{\alpha-2} P_{\mathrm{t}}^{2} / 4 k_{\mathrm{B}}^{2}$,

$\Gamma P_{\mathrm{c}} V_{\mathrm{c}}=\Gamma P_{\mathrm{c} 0} V_{\mathrm{c} 0}\left(\frac{n_{\mathrm{c}}}{n_{\mathrm{c} 0}}\right)^{\delta+1+\beta}=-\frac{\chi}{4 k_{\mathrm{B}}^{2}} T_{\mathrm{t}}^{\alpha-2} L_{\mathrm{t}} P_{\mathrm{t}}^{2}$,

where the power-law relationships for $P_{\mathrm{c}}$ and $V_{\mathrm{c}}$ (Eqs. (8) and (11), respectively) are used. Recalling that $\beta=\delta(\alpha-1)+1$, 
then the power of $n_{\mathrm{c}}$ on the left-hand side of Eq. (15) must be $\delta \alpha+2$.

Given the scaling of the left-hand side of the equation with $n_{\mathrm{c}}$, then the transition region radiative energy loss must also exhibit the same dependence on $n_{\mathrm{c}}$, which can be expressed via power-law relationships. More specifically, we require power-law relationships, with respect to $n_{\mathrm{c}}$, for the quantities $P_{\mathrm{t}}$ and $L_{\mathrm{t}}$. The necessary relationships can be obtained using straightforward physical reasoning.

\section{Transition region pressure}

It was stated above that the down-flow arises from a weakening of the pressure gradient between the transition region and the corona, rendering it unable to support the plasma against gravity in hydrostatic equilibrium. Therefore, as a first approximation we will assume that $P_{\mathrm{t}} \approx P_{\mathrm{c}}$ :

$P_{\mathrm{t}}=P_{\mathrm{c} 0}\left(\frac{n_{\mathrm{c}}}{n_{\mathrm{c} 0}}\right)^{\delta+1}$.

\section{Transition region thickness}

As the corona radiatively cools its thermal energy decreases and so, consequently, does the heat flux supplied to the transition region via thermal conduction. The transition region temperature gradient responds to changes in the heat flux that it is required to carry by changes in its own temperature gradient; a reduced heat flux results in a weaker temperature gradient and thus a thicker transition region. We expect the thickness of the transition region to obey a power-law relationship of the form:

$L_{\mathrm{t}}=L_{\mathrm{t} 0}\left(\frac{n_{\mathrm{c}}}{n_{\mathrm{c} 0}}\right)^{\epsilon}$,

where it is anticipated that $\epsilon<0$.

Substituting the power-law relationships into Eq. (15) gives:

$\Gamma P_{\mathrm{c} 0} V_{\mathrm{c} 0}\left(\frac{n_{\mathrm{c}}}{n_{\mathrm{c} 0}}\right)^{\delta \alpha+2}=-\frac{\chi}{4 k_{\mathrm{B}}^{2}} L_{\mathrm{t} 0} P_{\mathrm{c} 0}^{2} T_{\mathrm{t}}^{\alpha-2}\left(\frac{n_{\mathrm{c}}}{n_{\mathrm{c} 0}}\right)^{\epsilon+2(\delta+1)}$.

The value of the index $\epsilon$ can now be calculated as:

$\epsilon=\delta(\alpha-2)$.

For the values $\alpha=-\frac{1}{2}$ and $\delta=2$ quoted above and from CMA95, Eq. (12) gives $\beta=-2$, and we find that $\epsilon=-5$. CMA95 also found that for $\alpha=-2$ then $\delta \approx 1$, and so $\beta=-2$ and $\epsilon=-4$.

Finally, by equating the coeffients in Eq. (18) we find:

$V_{\mathrm{c} 0}=-\frac{\chi}{4 \Gamma k_{\mathrm{B}}^{2}} L_{\mathrm{t} 0} P_{\mathrm{c} 0} T_{\mathrm{t}}^{\alpha-2}$,

and can substitute it into Eq. (13) in order to complete our expression for $\delta$ :

$\delta=\gamma-1+(\gamma+1)\left(\frac{L_{\mathrm{c}} n_{\mathrm{c} 0}^{2} T_{\mathrm{c} 0}^{\alpha}}{L_{\mathrm{t} 0} n_{\mathrm{t} 0}^{2} T_{\mathrm{t}}^{\alpha}}\right)$.

We recognise that the part of the second term in brackets is just the ratio of the initial radiative losses integrated over the coronal and transition region thicknesses respectively. For $\gamma=$ $5 / 3$ and $\delta=2$, this ratio must be 0.8 .
Table 1. Initial $(t=0)$ conditions for the numerical simulations of cooling loops.

\begin{tabular}{ccc}
\hline \hline Simulation & $L(\mathrm{Mm})$ & $T_{\text {apex }}(\mathrm{MK})$ \\
\hline 1 & 10 & 2 \\
2 & 40 & 2 \\
3 & 40 & 4 \\
\hline
\end{tabular}

Equations (12) and (19) show how the indices, for each of the power-law relationships that have been proposed, are related. Since we are interested primarily in $\delta$, the relationships between the indices can give us an idea of how the relevant physical processes must interact to control the evolution of the loop in order to give the robust scaling between $T$ and $n$.

If the power-law relationships are correct and the behaviour of the key parameters has been identified, then sensible values for $\alpha$ and $\delta$ (e.g. based upon observations and/or numerical simulations) should then yield sensible values for the other indices, and vice-versa.

In the following section we describe several numerical hydrodynamic simulations of cooling loops and from the results we calculate the values of the indices for each of the powerlaws that we have postulated. If our analytical model captures the true physical behaviour of a cooling loop then the values of the indices given above should be in reasonably good agreement with those calculated from the simulations, bearing in mind of course the approximations made in the above analysis.

\section{Comparison between the analytical model and numerical simulations}

The computational code HYDRAD (Bradshaw \& Mason 2003a,b; Bradshaw et al. 2004) was used to perform three simulations of cooling loops and different initial conditions were adopted for each one. These are summarised in Table 1. The initial conditions for each loop are an equilibrium between conductive and radiative losses, and a heating function. For a fixed length, a hotter loop has a higher density, and for a fixed temperature a shorter loop has a higher density (e.g. Rosner et al. 1978).

The HYDRAD code evolves the initial conditions in time by setting the volumetric heating rate to zero and solving the coupled mass, momentum and energy density equations that describe the hydrodynamics of a compressible plasma. The energy equation includes terms for convective transport, thermal conduction, energy gain (loss) by compression (rarefaction) and optically-thin radiation. HYDRAD calculates opticallythin radiation using data provided by the CHIANTI atomic database (Young et al. 2003), whereas many existing hydrodynamic models use a series of power-laws to reproduce the characteristic radiative loss vs. temperature curve for the solar atmosphere. While HYDRAD is capable of solving the equations of ionisation balance simultaneously with the hydrodynamic equations, in order to calculate the optically-thin radiation when the ion populations depart from their equilibrium values, in the present work we use the equilibrium values for 


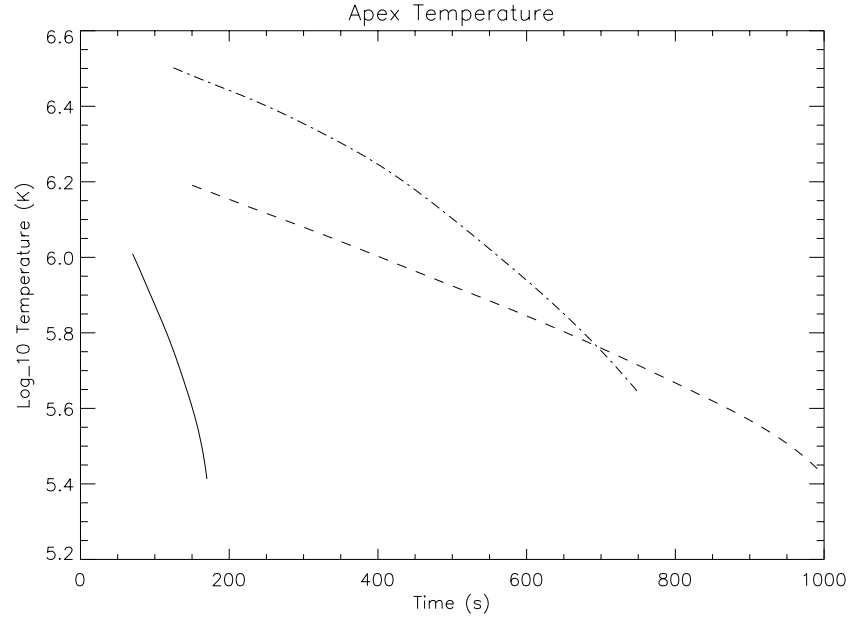

Fig. 1. The time-evolution of the apex temperature, during the radiative cooling phase, for simulation 1 (solid); simulation 2 (dashed); and simulation 3 (dot-dash).

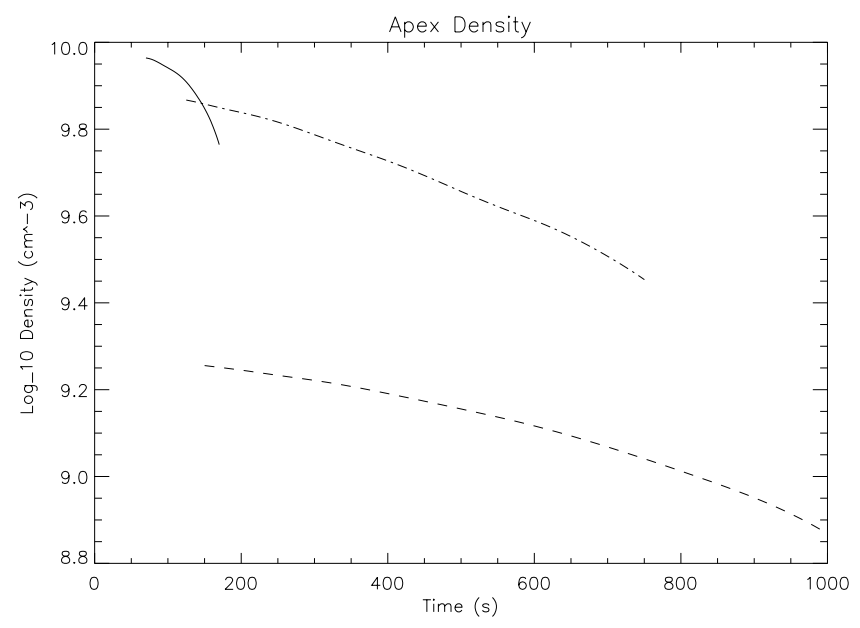

Fig. 2. The time-evolution of the apex density, during the radiative cooling phase, for simulation 1 (solid); simulation 2 (dashed); and simulation 3 (dot-dash).

the ion populations to retain compatibility with our analytical model.

HYDRAD also features an adaptive computational grid in order to ensure that small-scale and discontinuous features are captured with sufficient resolution as they propagate. Examples of such features are: shocks; thermal conduction fronts; and ionisation fronts. Since we have assumed that the energetics of the transition region control the dynamics of the loop, then it is extremely important that the transition region is captured with sufficient resolution in the case of a loop undergoing radiative cooling.

Figures 1 and 2 show the time-evolution of the temperature and the density at the loop apex during the radiative cooling phase of each simulation. The difference between the radiative energy losses at the base and apex of the loop have sufficiently weakened the pressure gradient so that the down-flow has been initiated. At the onset of the radiative phase it has become sufficient to drain plasma from the corona.

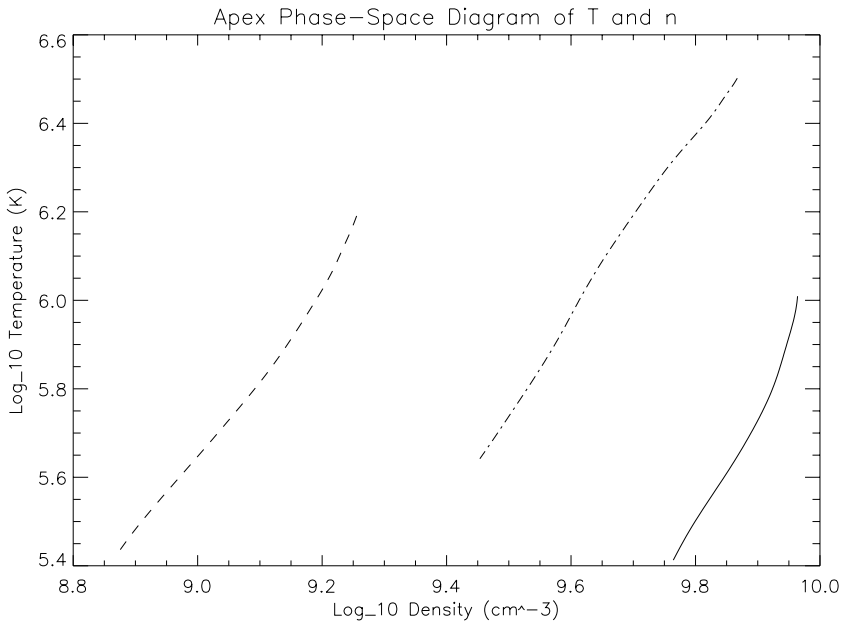

Fig. 3. Phase-space plots of temperature and density at the loop apex, during the radiative cooling phase, for simulation 1 (solid); simulation 2 (dashed); and simulation 3 (dot-dash). The gradient of each line gives the index $\delta$.

Of the two loops with an initial apex temperature of $2 \mathrm{MK}$, the shortest one cools the most rapidly. The difference in their lengths is a factor of 4 and the difference in their cooling timescales is a factor of 5.9, which suggests a weakly non-linear scaling between the cooling time-scale and the loop length of 1.28 , on the order of the $L^{5 / 6}$ scaling noted by Cargill (1993) and CMA95.

Of the two loops of length $40 \mathrm{Mm}$, the hotter one cools the most rapidly, reaching a temperature of $5 \times 10^{5} \mathrm{~K}$ at about $730 \mathrm{~s}$, which is on the order of one minute sooner than the other $40 \mathrm{Mm}$ loop. This cooling is consistent with the analytical models of Cargill (1993) and CMA95 who predict a relatively weak scaling of the cooling time with pressure, the relationship being $p^{-1 / 6}$.

Figure 3 shows the phase-space plots of $T$ and $n$ at the loop apex during the radiative cooling phase of each simulation. The gradient of each line gives the value of $\delta$ for the corresponding simulation. There is a clear power-law relationship between $T$ and $n$, and in each case $\delta \approx 2$, in agreement with previous findings (Sect. 1).

Figures 4 and 5 show the phase-space plots of the average values of $V_{\mathrm{c}}$ and $L_{\mathrm{t}}$, as functions of $n_{\mathrm{c}}$, during the radiative cooling phase, for each simulation. Calculating these average values is not a trivial matter since defining appropriate diagnostic measurements so that meaningful quantities can be extracted from numerical results can be very difficult. For example, how does one precisely and consistently define the transition region, bearing in mind that its location and properties change considerably during the simulations?

In order to calculate average values for the transition region properties $P_{\mathrm{t}}$ and $L_{\mathrm{t}}$, and to normalise their values to the initial values at the onset of the radiative cooling phase, we define the transition region as being in the temperature interval between $2 \times 10^{4}$ and $2 \times 10^{5} \mathrm{~K}$. We find that varying the width of this temperature interval, though it alters the calculated values of the indices, does not affect the generic conclusions that we draw from the numerical results. The average coronal 


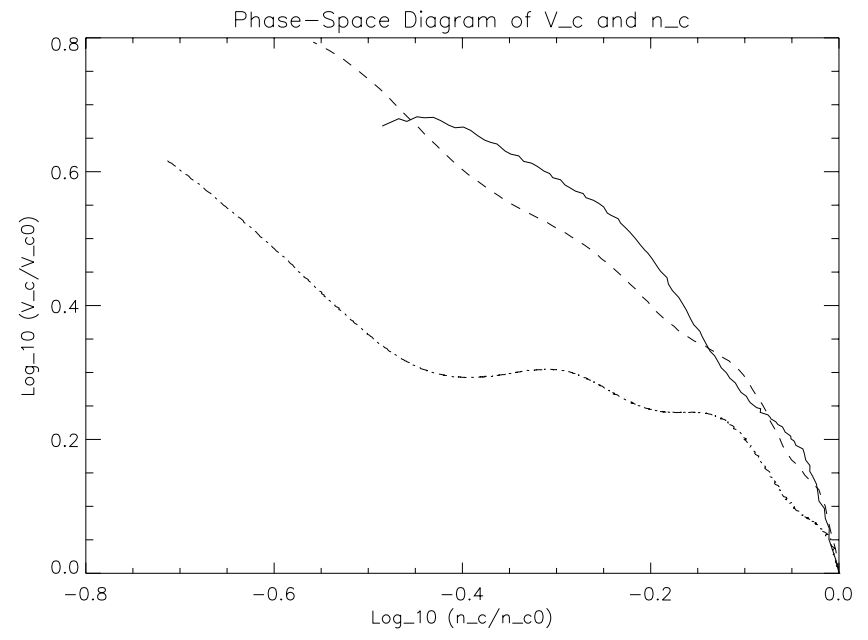

Fig. 4. Phase-space plots of $V_{\mathrm{c}}$ and $n_{\mathrm{c}}$ averaged along the coronal region of the loop, during the radiative cooling phase, for simulation 1 (solid); simulation 2 (dashed); and simulation 3 (dot-dash). The gradient of each line gives the index $\beta$.

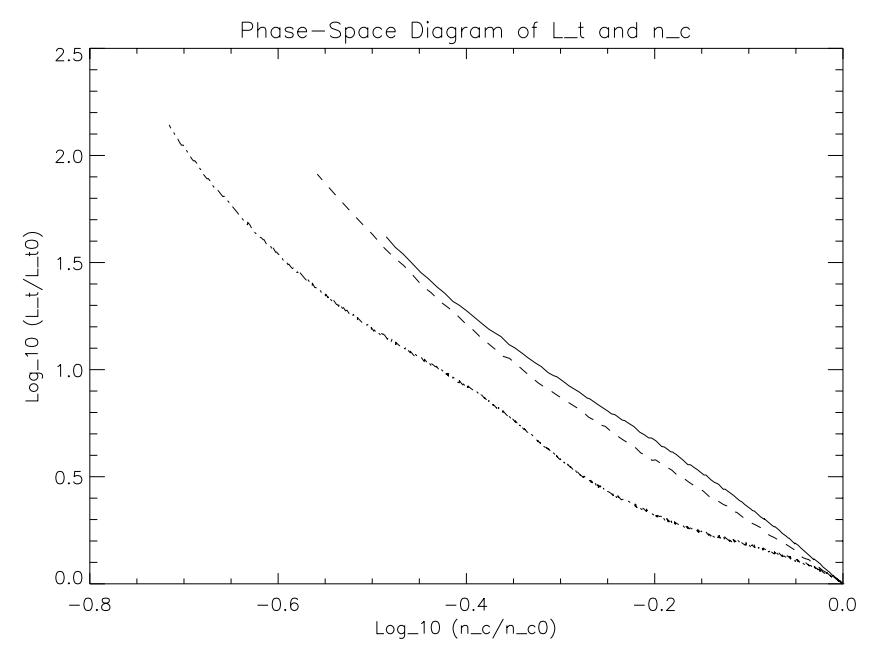

Fig. 5. Phase-space plots of $L_{\mathrm{t}}$ and $n_{\mathrm{c}}$ averaged along the transition and coronal regions of the loop, respectively, during the radiative cooling phase, for simulation 1 (solid); simulation 2 (dashed); and simulation 3 (dot-dash). The gradient of each line gives the index $\epsilon$.

values $\left(n_{\mathrm{c}}\right.$ and $\left.V_{\mathrm{c}}\right)$ are calculated between the top of the transition region and the loop apex.

Figure 4 shows the scaling relationship between $V_{\mathrm{c}}$ and $n_{\mathrm{c}}$ in each simulation. Although there is some variation of the scaling as the loop cools, $\beta$ is on average negative with average values between -2.0 and -0.9 . $\beta$ varies the most during simulation 3 , though it also does not remain absolutely constant during simulations 1 and 2 either. This indicates that the evolution of $V_{\mathrm{c}}$ is more complicated than a straightforward power-law can describe, however, the figures also show that it is reasonable to take an average value of $\beta$ in order to capture the overall behaviour of $V_{\mathrm{c}}$. Figure 5 shows a clear and consistent scaling relationship between $L_{\mathrm{t}}$ and $n_{\mathrm{c}}$ in each simulation, with $\epsilon$ in the range -3.5 to -3 .

Table 2 compares the values of the indices $\beta$ and $\epsilon$ predicted by the analytical model, with the average values calculated from the simulation results.
Table 2. Comparison between analytical predictions and numerical hydrodynamic calculations of the power-law indices $\beta$ and $\epsilon$.

\begin{tabular}{ccccc}
\hline \hline Index & Model & Sim. 1 & Sim. 2 & Sim. 3 \\
\hline$\beta$ & -2.0 & -2.0 & -1.5 & -0.9 \\
$\epsilon$ & -5.0 & -3.2 & -3.5 & -3.0 \\
\hline
\end{tabular}

The analytical prediction of $\beta=-2.0$ is in reasonable agreement with the numerical hydrodynamic calculations for simulations 1 and 2, though the value of -0.9 given by simulation 3 shows more deviation. The analytical prediction of $\epsilon=-5.0$ appears to be somewhat of an over-estimate in magnitude, since the numerical hydrodyanmic calculations yield reasonably similar values, between -3.0 and -3.5 .

Despite these differences, the predictions for the values of the indices made by the analytical model are encouraging, especially when one takes into account the averaging process used to calculate the indices and the more significant problem of the difficulty in defining appropriate diagnostic measurements, as discussed above. Overall, each set of indices is in sufficiently good agreement that a different choice of diagnostic would not lead us to significantly different conclusions. Furthermore, HYDRAD calculates the radiative emission using a full treatment of the atomic physics of the process, with data provided by CHIANTI (Young et al. 2003), rather than a simple scaling with $T^{\alpha}$. Since we have related $\beta$ and $\epsilon$ to $\alpha$, it is to be expected that the analytical and numerical values for the indices are not in exact agreement.

However, the main and most crucial finding is that our analytical model not only provides reasonable values for each of the indices, it describes in a simple way the overall behaviour of the physical system. This lends strong support to our assumption that thermal conduction everywhere along the loop, and convection in the transition region, can be neglected from the analytical model, consequently showing that optically-thin radiation from the transition region controls the evolution of the loop during the radiative cooling phase and establishes the robust power-law relationship between $T$ and $n$.

Some observational support for these scalings comes from the work of Sylwester et al. (1993). They first carried out numerical hydrodynamic simulations of cooling flare plasma and from the results calculated $n-T$ and corresponding $\sqrt{E M}-T$ diagrams, where $E M$ is the emission measure. For $\delta \approx 1.96$ they found $\delta_{E M} \approx 1.46$, i.e. $T \propto(\sqrt{E M})^{1.46}$. Following this, they used the calcium spectrum of a selection of flares recorded by the Solar Maximum Mission Bent Crystal Spectrometer to analyse the relationship between $E M$ and $T$, and found the observationally derived value $\delta_{E M} \approx 1.32$. Though not conclusive, these results are indicative of the presence of these scalings.

\section{An improved analytical model}

The analytical model presented in Sect. 2 makes good predictions of the power-law relationships between key variables during the radiative cooling phase. However, an improved treatment of the physics should provide even better predictions and, in particular, we address the over-estimation of the magnitude 


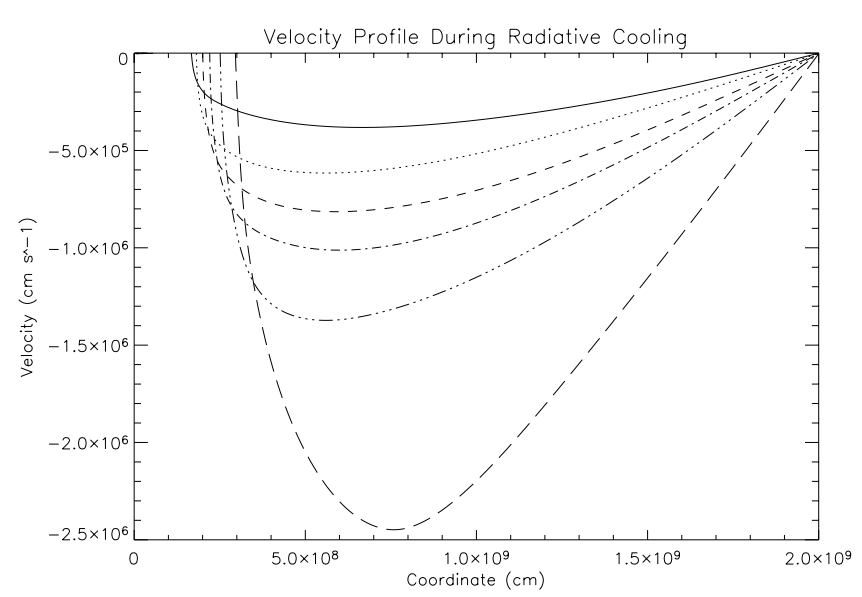

Fig. 6. Time series of the velocity profile between the loop base and apex, during the radiative cooling phase, for simulation 2 at: $150 \mathrm{~s}$ (solid line); $300 \mathrm{~s}$ (dotted); $450 \mathrm{~s}$ (dashed); $600 \mathrm{~s}$ (dot-dash); $800 \mathrm{~s}$ (triple-dot-dash); $1000 \mathrm{~s} \mathrm{(long-dashed).}$

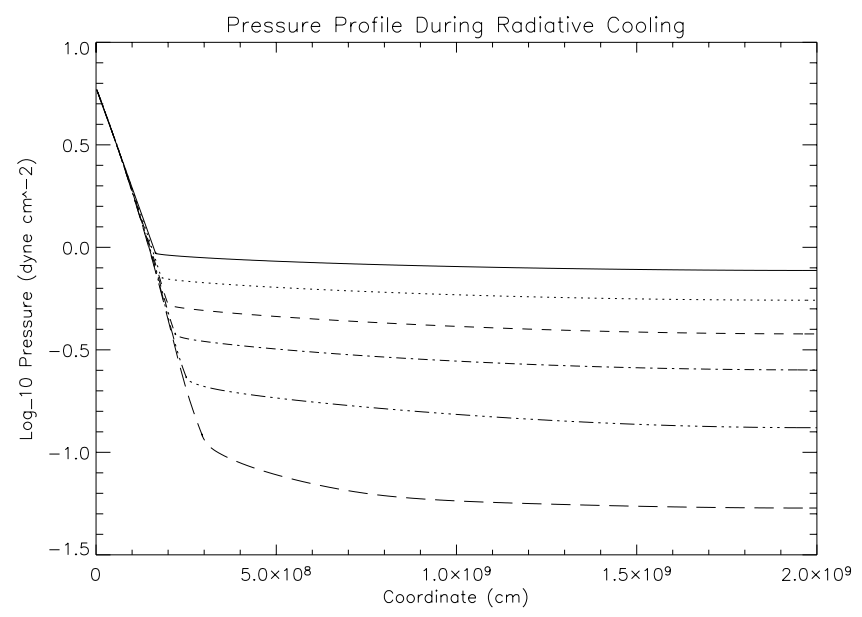

Fig. 7. Time series of the pressure profile between the loop base and apex, during the radiative cooling phase, for simulation 2 at: $150 \mathrm{~s}$ (solid line); $300 \mathrm{~s}$ (dotted); $450 \mathrm{~s}$ (dashed); $600 \mathrm{~s}$ (dot-dash); $800 \mathrm{~s}$ (triple-dot-dash); $1000 \mathrm{~s}$ (long-dashed).

of the index $\epsilon$ that described the thickness of the transition region.

We stated previously that the down-flow associated with radiative cooling arises due to a weakening of the pressure gradient that supports the plasma against gravity in hydrostatic equilibrium. Figure 6 shows how the down-flow develops during simulation 2 and Fig. 7 shows the corresponding pressure profile along the loop.

Figure 7 shows that the pressure monotonically increases towards the loop base (i.e. $P_{\mathrm{t}}>P_{\mathrm{c}}$ ). Therefore, the pressure gradient cannot contribute towards driving the down-flow but, rather, acts to oppose it. However, the pressure gradient is not sufficient to halt the down-flow altogether and so it must infact regulate or attenuate the down-flow against the downward gravitational force exerted by the Sun.

With this in mind we now drop the assumption that $P_{\mathrm{t}} \approx$ $P_{\mathrm{c}}$ in our original model and adopt a new formulation for $P_{\mathrm{t}}$

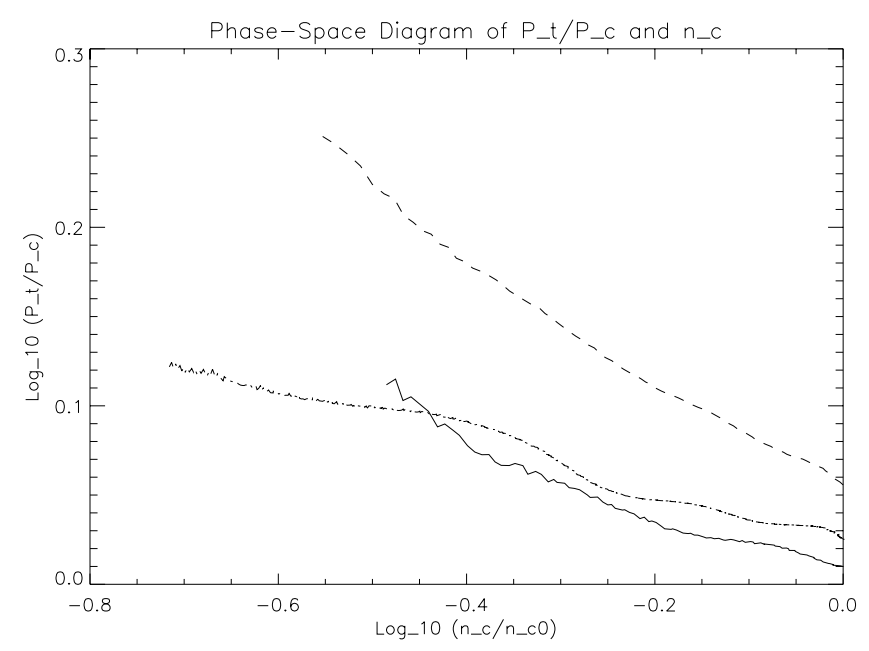

Fig. 8. Phase-space plots of $\frac{P_{\mathrm{t}}}{P_{\mathrm{c}}}$ and $n_{\mathrm{c}}$ averaged along the transition and coronal regions of the loop, respectively, during the radiative cooling phase, for simulation 1 (solid), simulation 2 (dashed) and simulation 3 (dot-dash). The gradient of each line gives the index $\theta$.

in order to account for the presence, as shown by Fig. 7, of a pressure gradient between the corona and the transition region:

$P_{\mathrm{t}}=K P_{\mathrm{c}}$,

where $K$ is small and on the order of 1 . Let:

$K=\left(\frac{n_{\mathrm{c}}}{n_{\mathrm{c} 0}}\right)^{\theta}$,

thus, from Fig. 7, we expect $\theta$ to be small and negative since $n_{\mathrm{c}}<n_{\mathrm{c} 0}$ as the radiative cooling proceeds. Now:

$P_{\mathrm{t}}=P_{\mathrm{c} 0}\left(\frac{n_{\mathrm{c}}}{n_{\mathrm{c} 0}}\right)^{\delta+\theta+1}$.

Substituting Eqs. (24) and (17) into Eq. (15) gives:

$\alpha \delta+2=\epsilon+2(\delta+\theta+1)$.

Figure 8 shows the phase-space plots of the average values of $\frac{P_{\mathrm{t}}}{P_{\mathrm{c}}}$, as functions of $n_{\mathrm{c}}$, during the radiative cooling phase for each simulation. The values of $\theta$ derived from the plots are: simulation $1, \theta=-0.2$; simulation $2, \theta=-0.4$; and simulation $3, \theta=-0.1$.

In Table 2 the value of $\epsilon$ given by the analytical model, which does not take into account the pressure gradient, is compared with the values given by the simulations, which obviously do account for the pressure gradient. Therefore, a better comparison could be made if the values of $\epsilon$ given by the simulations could be corrected, such that the effect of the pressure gradient is removed from the result.

From Eq. (19):

$\epsilon=\delta(\alpha-2)$,

and from Eq. (25):

$\epsilon=\delta(\alpha-2)-2 \theta$.

Therefore:

$\epsilon=\epsilon_{0}-2 \theta$, 
where $\epsilon_{0}$ is the value obtained when the pressure gradient has not been accounted for. Equation (28) provides a way to correct the values of $\epsilon$ for each simulation to give an estimate of $\epsilon_{0}$, which can be compared more directly with the analytical estimate of -5.0 . The values given by the simulations are: simulation 1, $\epsilon_{0}=-3.6$; simulation $2, \epsilon_{0}=-4.3$; and simulation 3 , $\epsilon_{0}=-3.2$. Each of these values is in better agreement with the analytical estimate, as a consequence of developing a more accurate treatment of the key physics.

The discrepancies between our analytical and numerical models, in the values for the power-law indices, are due to the treatment of optically-thin radiation from the transition region. The analytical model necessarily employs a very simplistic treatment of the form $E_{\mathrm{rad}} \propto \chi T^{\alpha}$ (e.g. Priest 1982), which is a function of temperature only, whereas HYDRAD uses data from the CHIANTI atomic database (Young et al. 2003) to provide a far more accurate and comprehensive calculation of the radiative emission as a function of both temperature and density. Cargill et al. (1995) also demonstrated the sensitivity of the value of $\delta$ to the value chosen for $\alpha$. The differences between the values for the power-law indices are also due partly to the difficulties in defining appropriate and consistent definitions for transition region diagnostics, as discussed in Sect. 3.

\section{Summary and conclusions}

We have developed an analytical model of the radiative cooling phase of a coronal loop in order to establish the physical mechanism behind the robust $T \propto n^{\delta}$ scaling. Our analytical model assumes that during the radiative cooling phase the corona loses energy by radiation and, due to the presence of a down-flow, by convection to the transition region; the energy supplied to the transition region by the corona is radiated away to space.

We have shown, through the use of additional power-law relationships, that radiative emission from the transition region controls the evolution of the loop during the radiative phase. This has been achieved by comparing the scaling with coronal density of the average coronal down-flow velocity and the thickness of the transition region as predicted by our analytical model, with the scaling given by full, numerical hydrodynamic simulations. Given the approximations used in our analytical model and the difficulty of defining appropriate diagnostic measurements, we have found remarkably good agreement between the values of the indices of the power-laws predicted by the analytical model and those given by the simulations. Since the simulations solve a full energy equation, including terms that account for convection, thermal conduction, compressibility and radiation, and only radiation is considered in the analytical model, the agreement between the predicted values for the indices demonstrates conclusively that transition region radiation is the dominant physical process governing the evolution of the loop during the radiative cooling phase and gives rise to the robust $T \propto n^{\delta}$ scaling.

We have also shown that the down-flow associated with radiative cooling is initiated by a weakening of the pressure gradient between the corona and the transition region, due to differences in the radiative emission, so that the plasma can no longer be supported in hydrostatic equilibrium. Hence, rather than driving the down-flow, the pressure gradient actually serves to regulate it and acts as a brake to gravitational acceleration. We demonstrated this by accounting for the presence of a pressure gradient in our analytical model and showing how a correction can be derived to provide a more direct comparison between the indices predicted by the analytical model and those given by the simulations.

Acknowledgements. S.J.B. acknowledges support from a PPARC Rolling Grant. P.J.C. acknowledges the support of a PPARC senior research fellowship.

\section{References}

Bradshaw, S. J., Del Zanna, G., \& Mason, H. E. 2004, A\&A, 425, 287 Bradshaw, S. J., \& Mason, H. E. 2003a, A\&A, 401, 699

Bradshaw, S. J., \& Mason, H. E. 2003b, A\&A, 407, 1127

Cargill, P. J. 1993, Sol. Phys., 147, 263

Cargill, P. J. 1994, ApJ, 422, 381

Cargill, P. J., \& Klimchuk, J. A. 2004, ApJ, 605, 911

Cargill, P. J., Mariska, J. T., \& Antiochos, S. K. 1995, ApJ, 439, 1034 Jakimiec, J., Sylwester, B., Sylwester, J., et al. 1992, A\&A, 253, 269

Priest, E. R. 1982, Solar magneto-hydrodynamics (Dordrecht, Holland, Boston: D. Reidel Pub. Co., Hingham)

Rosner, R., Tucker, W. H., \& Vaiana, G. S. 1978, ApJ, 220, 643

Serio, S., Reale, F., Jakimiec, J., Sylwester, B., \& Sylwester, J. 1991, A\&A, 241, 197

Sylwester, B., Sylwester, J., Serio, S., et al. 1993, A\&A, 267, 586

Young, P. R., Del Zanna, G., Landi, E., et al. 2003, ApJS, 144, 135 